\title{
Slow light and chromatic temporal dispersion in photonic crystal waveguides using femtosecond time of flight
}

\author{
C. E. Finlayson, ${ }^{*}$ F. Cattaneo, N. M. B. Perney, and J. J. Baumberg ${ }^{\dagger}$ \\ School of Physics \& Astronomy, University of Southampton, Southampton SO17 1BJ, United Kingdom \\ M. C. Netti, M. E. Zoorob, and M. D. B. Charlton \\ Mesophotonics Ltd, Chilworth Science Park, Southampton, SO16 7NP, United Kingdom \\ G. J. Parker \\ School of Electronics \& Computer Science, University of Southampton, Southampton SO17 1BJ, United Kingdom
}

(Received 21 October 2005; published 30 January 2006)

\begin{abstract}
We report time-of-flight experiments on photonic-crystal waveguide structures using optical Kerr gating of a femtosecond white-light supercontinuum. These photonic-crystal structures, based on engineered siliconnitride slab waveguides, possess broadband low-loss guiding properties, allowing the group velocity dispersion of optical pulses to be directly tracked as a function of wavelength. This dispersion is shown to be radically disrupted by the spectral band gaps associated with the photonic-crystal periodicity. Increased time-of-flight effects, or "slowed light," are clearly observed at the edges of band gaps in agreement with two-dimensional plane-wave theoretical models of group velocity dispersion. A universal model for slow light in such photonic crystals is proposed, which shows that slow light is controlled predominantly by the detuning from, and the size of, the photonic band gaps. Slowed light observed up to time delays of $\sim 1 \mathrm{ps}$, corresponds to anomalous dispersion of $\sim 3.5 \mathrm{ps} / \mathrm{nm}$ per $\mathrm{mm}$ of the photonic crystal structure. From the decreasing intensity of timegated slow light as a function of time delay, we estimate the characteristic losses of modes which are guided in the spectral proximity of the photonic band gaps.
\end{abstract}

DOI: 10.1103/PhysRevE.73.016619

PACS number(s): 42.70.Qs, 42.65.-k, 42.79.-e

\section{INTRODUCTION}

Photonic crystals (PC's) are one-, two- or threedimensional periodic structures with the ability to confine, control, and modify the flow of light into discreet energy bands and to localize optical fields into very small volumes of space [1-3]. In addition to the extensive theoretical modeling of such structures, practical demonstrations involving variations in dielectric permittivity with periodicity, or quasiperiodicity, of similar length scale to the wavelength of light have also been widely investigated. One-dimensional photonic crystals have been well known and understood for many decades, an example being the Bragg mirror which is nowadays employed in a wide range of optical and optoelectronic applications. Two- and three-dimensional (2D and 3D) photonic structures are more difficult to achieve, since the required features are at the limits, or go beyond the current limitations, of conventional lithography and etching techniques, whereby material is removed from a host medium, producing thin films which are periodically patterned between the material and air [4-6]. One notable variation to these techniques is the use of self-assembly and electrochemical deposition techniques to produce arrays of threedimensional photonic patterns [7]. Imperfections in the patterning cause diffraction losses and, most importantly, can

\footnotetext{
*Currently at Cavendish Laboratory, University of Cambridge, CB3 0HE, UK.

${ }^{\dagger}$ Electronic mail: j.j.baumberg@soton.ac.uk
}

reduce the effects of coherent scattering which is the basis for the formation of photonic band gaps. The production of high-quality 2D and 3D photonic crystals, with optimized designs, therefore represents a very critical challenge. The ability to control optical propagation by patterning planar devices in a single microfabrication step makes $2 \mathrm{D}$ photoniccrystal waveguides, in particular, a very attractive platform for realizing integrated optical circuits [8] in a wide range of dielectric and semiconductor material systems $[9,10]$. The functionality of different elements can be intricately engineered by varying the local symmetry and geometry of the PC lattice $[11,12]$, allowing modification of the photon dispersion relations and, ultimately, to a tailoring of group velocity dispersion (GVD), photonic band gaps, localized states, and enhancement of nonlinear effects [13]. Yet further functionality may be achieved by the introduction of disordered "defect states" into the structure [14] and by modification of the spontaneous emission lifetime of embedded emitters $[15,16]$, for example.

The spectral and spatial characteristics of PC's, and the correlation to theoretical models, have been widely studied experimentally. However, there are relatively few reported measurements of how light actually propagates through PC's in the temporal domain. The measurement and characterization of the GVD of optical pulses as they propagate in a PC are crucial to the demonstration of useful dispersive properties. One particularly interesting theoretically predicted property is the anomalous behavior of GVD in the spectral regions immediately adjacent to photonic band gaps (PBG's) $[17,18]$. In a similar fashion to the strong lattice interaction 
(a)

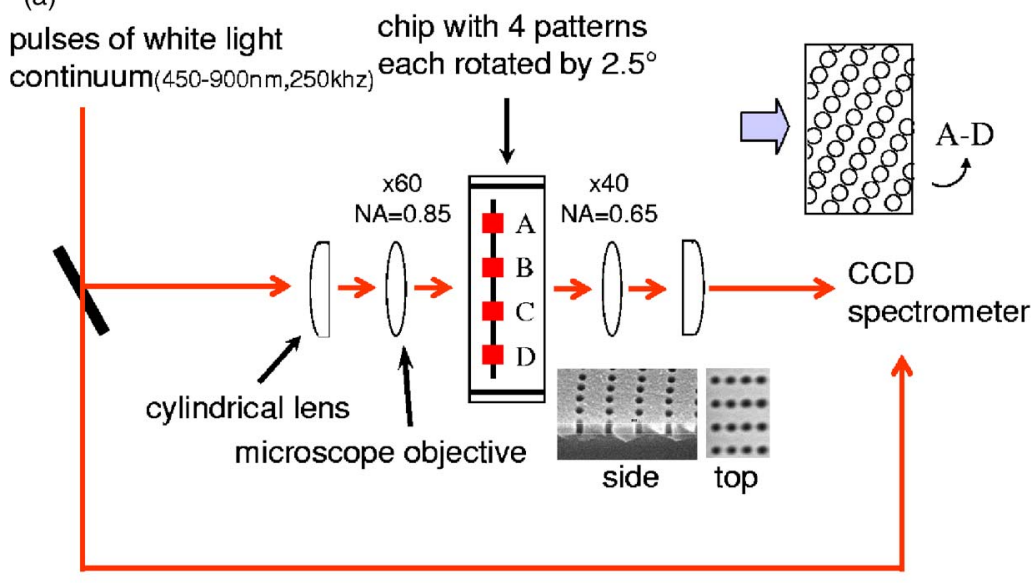

(b)

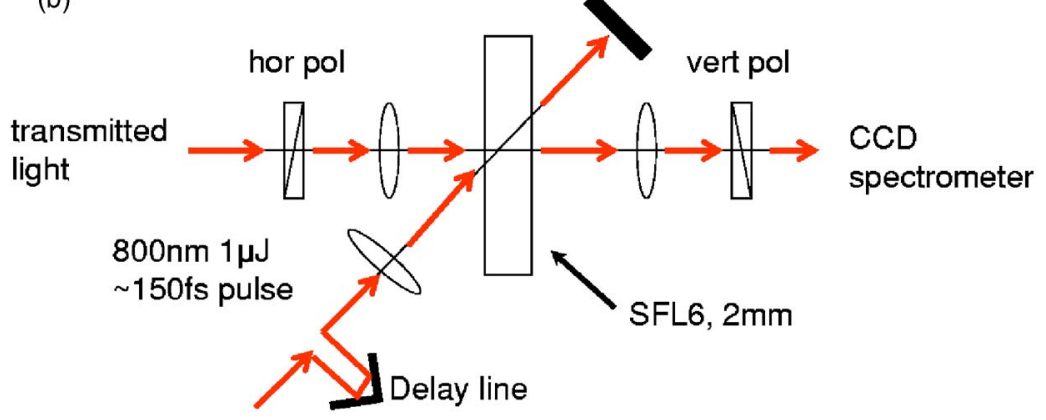

FIG. 1. (Color online) (a) Schematic of the experimental transmittance setup, showing the tight focusing of the broadband white-light continuum though the sample waveguide, in a perpendicular direction to the front facet of the waveguide and the front interface of the photonic crystal (PC) patterns $A-D$. The insets display scanning electron microscope (SEM) images of the PC patterns, in both side-on and top view, and also a schematic of the pattern rotation relative to the probe propagation direction. (b) Schematic setup for time-of-flight experiments; transmitted light from the sample passes through an optical Kerr gate to allow temporal resolution of different components of the white-light continuum, while varying the time delay of the gating pulses. of electronic states close to the Brillouin zone boundary in a semiconductor, it is expected that the group velocity of light in the vicinity of the PBG will be sharply reduced, becoming asymptotically equal to zero within the band gap. The result of this GVD anomaly is the "slowing down" of light close to the PBG, with clear implications for the design of PC dispersion compensators [19] and optical circuits. Several groups have previously attempted to indirectly extract the GVD using various interferometric and autocorrelation techniques [20-23] or by using near-field scanning techniques to probe the position in real space of optical pulses [24,25]. However, unambiguous experimental observation of such GVD anomalies remains to be demonstrated.

In this paper, we report time-of-flight experiments on photonic-crystal waveguide structures using a femtosecond white-light supercontinuum and an optical Kerr gate [26,27]. Broadband transmission measurements on our structures show them to possess very low loss guiding properties. As reported in our previous study of modal dispersion and birefringence engineering in slab optical waveguides [28], these techniques allow us to directly observe the propagation of ultrafast pulses of light through the structures, with a temporal resolution of around $250 \mathrm{fs}$, and do not present any difficulties of phase-matching requirements between pump and probe. We have clearly observed signatures of the anticipated "slowed-light" effects close to the photonic band gaps of our samples and have measured and characterized these effects in detail, with the aid of suitable 2D plane-wave theoretical models [29] of the photonic band-structure and GVD.

\section{EXPERIMENT}

The slab waveguide samples investigated in these experiments were constructed from silicon-nitride $\left(\mathrm{Si}_{3} \mathrm{~N}_{4}\right)$ core layers $(n=2.02)$, embedded in silicon-dioxide $\left(\mathrm{SiO}_{2}\right)$ substrate and cladding layers $(n=1.46)$, the whole structure being grown on silicon by low-pressure chemical vapor deposition (LPCVD). The nominal thickness of the layers was $2100 \mathrm{~nm}$ (substrate), $175 \mathrm{~nm}$ (core), and $200 \mathrm{~nm}$ (cladding).

The waveguides were then patterned with a $2 \mathrm{D}$ photonic crystal, using reactive ion-beam etching. In order to transfer the desired pattern into the waveguide layer it is necessary to first deposit a layer of chrome, followed by a further layer of positive photoresist $[6,30]$. The pattern is written into the photoresist with an electron beam and then transferred into the chrome layer using a wet-chrome etch and, finally, into the waveguide using reactive ion etching, down to a depth of $500 \mathrm{~nm}$. Patterns consisted of engineered arrays of triangular, square, or rectangular arrangements with period (or pitch) from 260 to $410 \mathrm{~nm}$, hole diameter from 160 to $250 \mathrm{~nm}$ (hole aspect ratios, hence, of 2-3), and number of repeating rows from 150 to 600 . Samples contained four patterns rotated, in steps of $2.5^{\circ}$ with respect to each other, in order to probe different directions of propagation through the sample structure [see Fig. 1(a)]. The patterns were then finally capped with a thin layer of silica, in order to reduce the optical scattering properties of the waveguide structure.

The optical setup used to characterize the transmittance properties of our 2D photonic-crystal waveguide samples is shown in Fig. 1. A broadband, white-light continuum, de- 
rived by tightly focusing regenerative amplified $\sim 150$-fs pulses from a Ti:sapphire laser $(\lambda=800 \mathrm{~nm}, 1 \mu \mathrm{J})$ into a sapphire crystal, was used as the probe source. The well-known combination of nonlinear effects results in a broad shortpulse continuum, ranging from around $\lambda=500 \mathrm{~nm}$ to $>1 \mu \mathrm{m}$, with a fairly uniform intensity profile as a function of wavelength. This white-light beam is then divided into two using a beam splitter near normal incidence; one part is sent to the sample stage and is tightly focused through the waveguide samples using a system of cylindrical and spherical lenses (Fig. 1), with the other being routed independently into the detector as a reference beam in order to facilitate the determination of the relative transmittance of the sample. Prior to being coupled through the experimental setup, the continuum is passed through an optical half-wave plate to allow independent analysis of the TE and TM polarizations. The two signals are spectrally analyzed using a highresolution spectrometer with a nitrogen-cooled chargecoupled-device (CCD) detector. The samples were carefully horizontally translated, in the direction perpendicular to the probe light beam, in order to complete the measurement of each of the four PC patterns within the set.

The light transmitted by the samples was also characterized in the time domain with the use of an optical Kerr shutter, allowing information about the temporal dispersion of the probe pulse due to the waveguide and photonic structure to be directly measured. This optical shutter is constructed by routing the transmitted light through a system of two crossed optical polarizers and by placing a 2-mm-thick glass slab (SFL6, with a high Kerr coefficient [27]) in between them, into which the light is focused. The optical shutter is opened by focusing intense optical pulses, also from the regeneratively amplified Ti:sapphire laser $(\sim 150$ fs pulse length, $\lambda$ $=800 \mathrm{~nm}, 1 \mu \mathrm{J}$ ), onto the glass at the same spatial position as the transmitted light. Due to the high third-order nonlinear susceptibility of SFL6, this "gate" pulse introduces a transient birefringence in the glass, which acts to partially rotate the polarization of the transmitted light, allowing partial transmission by the second polarizer. The arrival of the probe and gate pulses also must be correctly synchronized for the Kerr gate to operate. As the Ti:sapphire pulses used in the generation of the white-light continuum probe and in the Kerr gate operation are from a common source, it is possible to achieve synchronization by using a delay line on the gating pulse that adjusts the optical path difference between probe and gate in order to time-resolve the signal. An illustration of the optical setup used in these time-of-flight measurements is shown in Fig. 1(b).

By varying the gate delay in suitable increments, it is hence possible to directly observe the "time of flight" of different wavelength components of the continuum using a spectrometer and, by inference, to map out the GVD characteristics of the sample. This technique has many advantages over other nonlinear methods (such as up-conversion and frequency mixing) [31], as there are no phase-matching requirements in the Kerr shutter allowing a broadband range of wavelengths and numerical apertures from the waveguide to be analyzed.

The probe light which is transmitted by the Kerr gate is a convolution of the intensity of the probing and gated pulses according to the formula

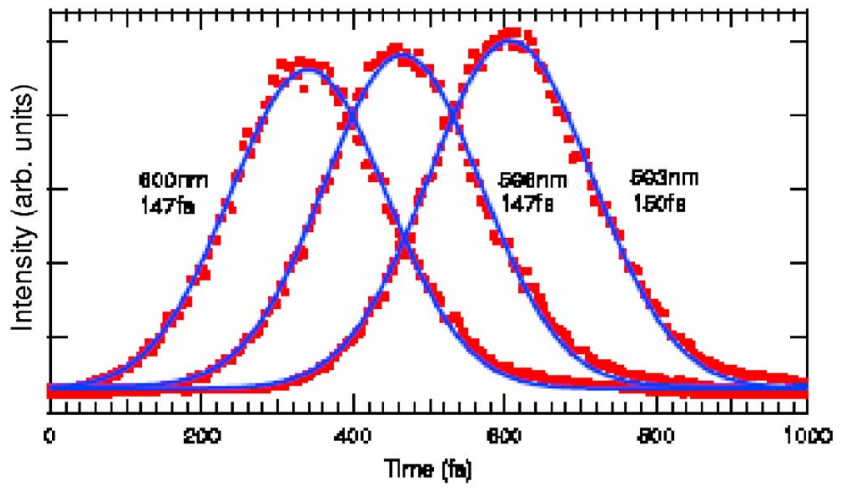

FIG. 2. (Color online) Measured temporal resolution of the Kerr gating technique, from the time-of-flight dispersion of the whitelight continuum, in the absence of a sample.

$$
T(t, \lambda) \propto \int_{-\infty}^{+\infty} I_{\text {probe }}(\tau, \lambda) I_{\text {gate }}^{2}(\tau-t) d \tau,
$$

where $I_{\text {probe }}$ and $I_{\text {gate }}$ are the intensity of the probing and gating pulses, respectively. It can be seen from this that the gate efficiency and, hence, the detected signal intensity are dependent on the square of the gate pulse intensity. Careful optimization of the gate focusing conditions, as well as the gate-probe spatial overlap, is therefore a critical issue. In practice, we are able to achieve a maximum Kerr gate efficiency of the order of a few percent, before continuum generation from the gate pulse in the Kerr shutter medium masks the signal. By measuring the time-of-flight dispersion of the continuum, in the absence of a sample, in a series of very short time-delay increments and by considering the gated intensity for a fixed wavelength as a function of time, we were also able to measure the temporal resolution of our experiment, as shown in Fig. 2. Measurement of this resolution at several different wavelengths shows a consistent value of around $250 \mathrm{fs}$, with an almost instantaneous response. We can consider that this value represents a convolution of the gate pulse duration, of $150 \mathrm{fs}$, and the temporal persistence of the Kerr effect in the SFL6 medium.

\section{TRANSMITTANCE MEASUREMENTS}

The particular PC waveguide sample which was used for the experiments reported in this paper was a 150-row rectangular lattice, of pitch $a=310 \mathrm{~nm}, b=465 \mathrm{~nm}$, and hole diameter $160 \mathrm{~nm}$. The measured transmittance spectra (logarithmic scale) in the TM polarization, at $\lambda=500-800 \mathrm{~nm}$, for this sample are shown in Fig. 3, with the spectra $A, B, C$, and $D$ being those for structures with the $\Gamma-J$ axis rotated by $0^{\circ}$, $2.5^{\circ}, 5^{\circ}$, and $7.5^{\circ}$, respectively, from the direction perpendicular to the probe propagation. Three intense PBG features are observed, with extinction factors of at least two orders of magnitude, located in the case of structure $A$ at $\lambda=600 \mathrm{~nm}$, $620 \mathrm{~nm}$, and $730 \mathrm{~nm}$, and with a spectral width of $\sim 10 \mathrm{~nm}$. As the structure is rotated, $A-D$, we see the latter of these features shift systematically to the blue, while the two other features show a tendency to diverge in wavelength. In the case of structure $D$, we see the effects of a close spectral 


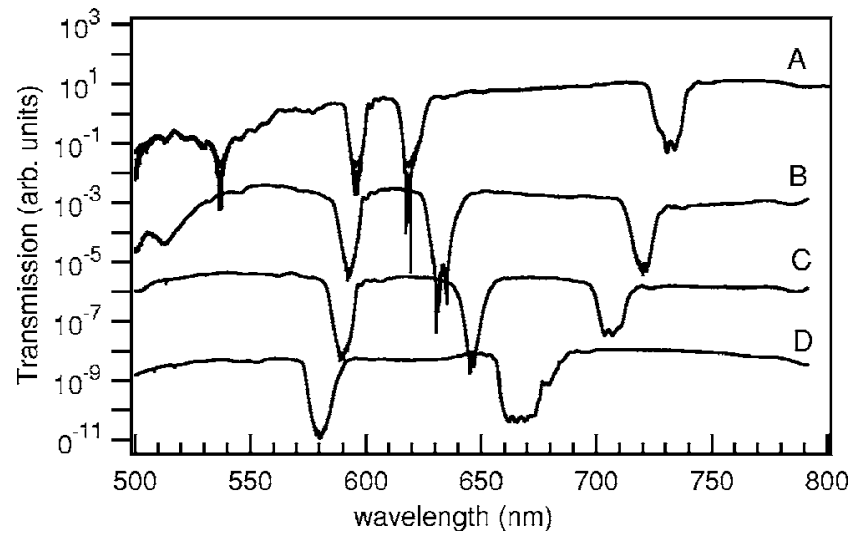

FIG. 3. Transmittance of a PC waveguide sample consisting of 150 rows rectangular lattice, of pitch $a=310 \mathrm{~nm}, b=420 \mathrm{~nm}$, and hole diameter $160 \mathrm{~nm}$. Spectra for TM polarization $A, B, C$, and $D$ are those for structures with the $\Gamma-J$ axis rotated by $0^{\circ}, 2.5^{\circ}, 5^{\circ}$, and $7.5^{\circ}$, respectively, from the direction of probe propagation. Spectra are also offset for clarity.

overlap between the two longer-wavelength features.

Of special note are the very low loss transmission properties of the samples, across virtually the entire visiblewavelength band. This is evident from the remarkably flat amplitude of the spectra, as referenced to the unpatterned waveguide which has transmission of order $100 \%$, at energies either side of the PBG features, not just on the lowenergy (dielectric) side of the band gap, but also on the highenergy (air) side, which is prone to losses due to upscattering out of the waveguide. Issues relating to guiding loss are amongst the key factors in the design and development of realistic PC waveguide structures in optoelectronic and integrated-optics devices [32,33], and these measurements compare very favorably with those previously reported.

In order to compare qualitatively our observed PBG features with those expected from a theoretical modeling of the photonic band structure, 2D plane-wave simulations for these particular PC structures were carried out and the results for the relevant TM polarization are shown in Fig. 4(a). This shows the dispersion relation (normalized frequency versus normalized wave vector) of the various dielectric- and airband modes which propagate through the structure, an important constraint being that only modes within the "light cone," bound by the speeds of light in the core and in the cladding layers, may propagate through the waveguide $[2,29]$. In theory, any intervals of frequency where these two criteria do not allow propagating modes will be characterized by zero transmittance (i.e., a photonic band gap). This analysis reveals that the three strong PBG features we see experimentally in the visible spectrum relate to gaps formed between the second dielectric band and the second air band, the third dielectric band and the third air band, and the fourth dielectric band and the fourth air band, respectively, in order of increasing frequency. Figure 4(b) shows how the predicted PBG features compare to those observed experimentally, and we see that the match is qualitatively satisfactory. Discrepancies between theory and experiment may be explained by the limitation of the 2D simulation, which does not take into account the finite extent of the sample in the third dimension, instead assuming the 2D PC lattice pattern to be infinite in extent. The adoption of more precise models, such as a quasi-3D plane-wave approach [34] or finite-difference time domain (FDTD) techniques [35], would involve a considerable increase in the computational complexity, beyond the scope of the current paper. As a further consequence of the limitations of the 2D model, we also note that, in general, the theoretically predicted PBG features are noticeably broader than those measured experimentally.

\section{TIME-OF-FLIGHT MEASUREMENTS}

Figure 5(a) illustrates the experimental time-of-flight data, for the PC waveguide sample described in the previous sec-
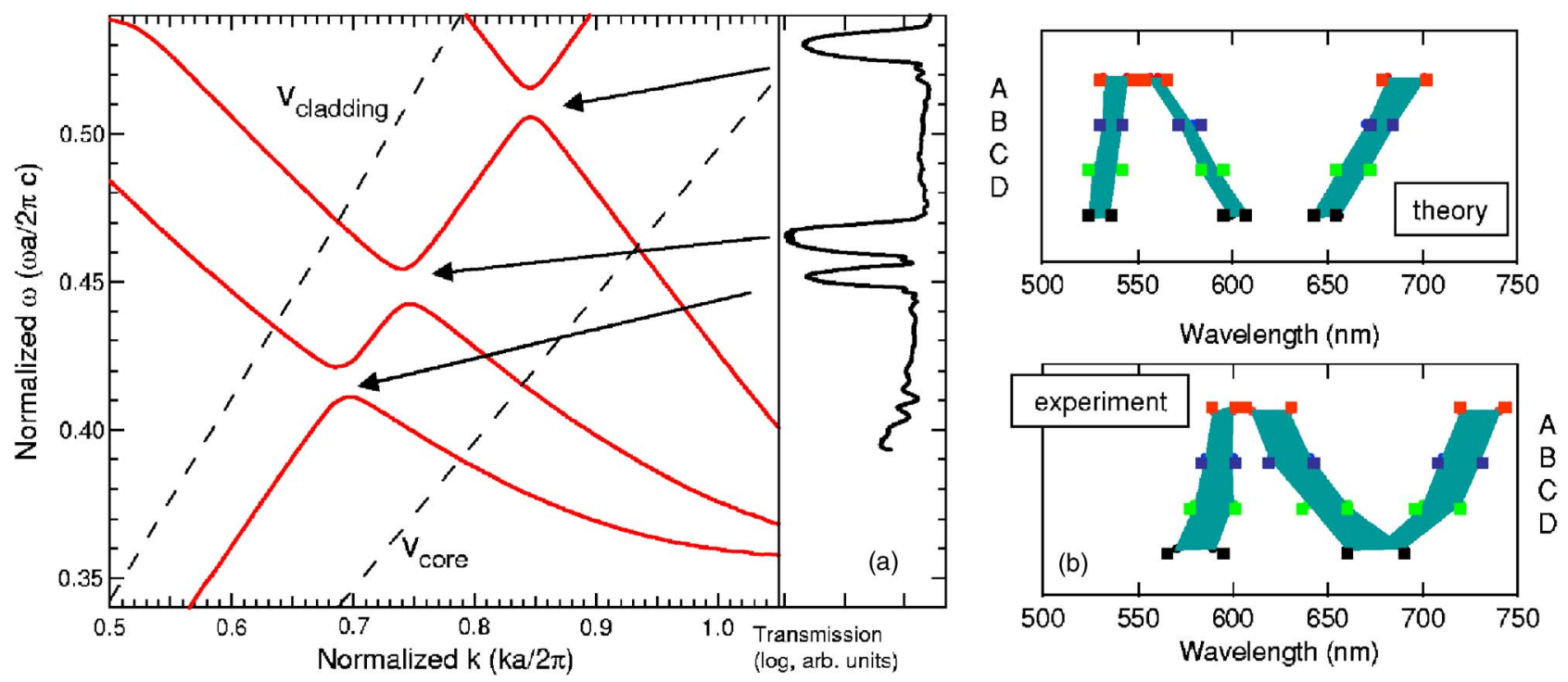

FIG. 4. (Color online) (a) Normalized band diagram from a 2D plane-wave simulation, for the photonic-crystal structure $D$ used in our experiments with the corresponding transmission spectrum. Dashed line show cladding and core light lines. (b) Comparison of theoretically predicted and experimental band gaps for structures $A-D$. 

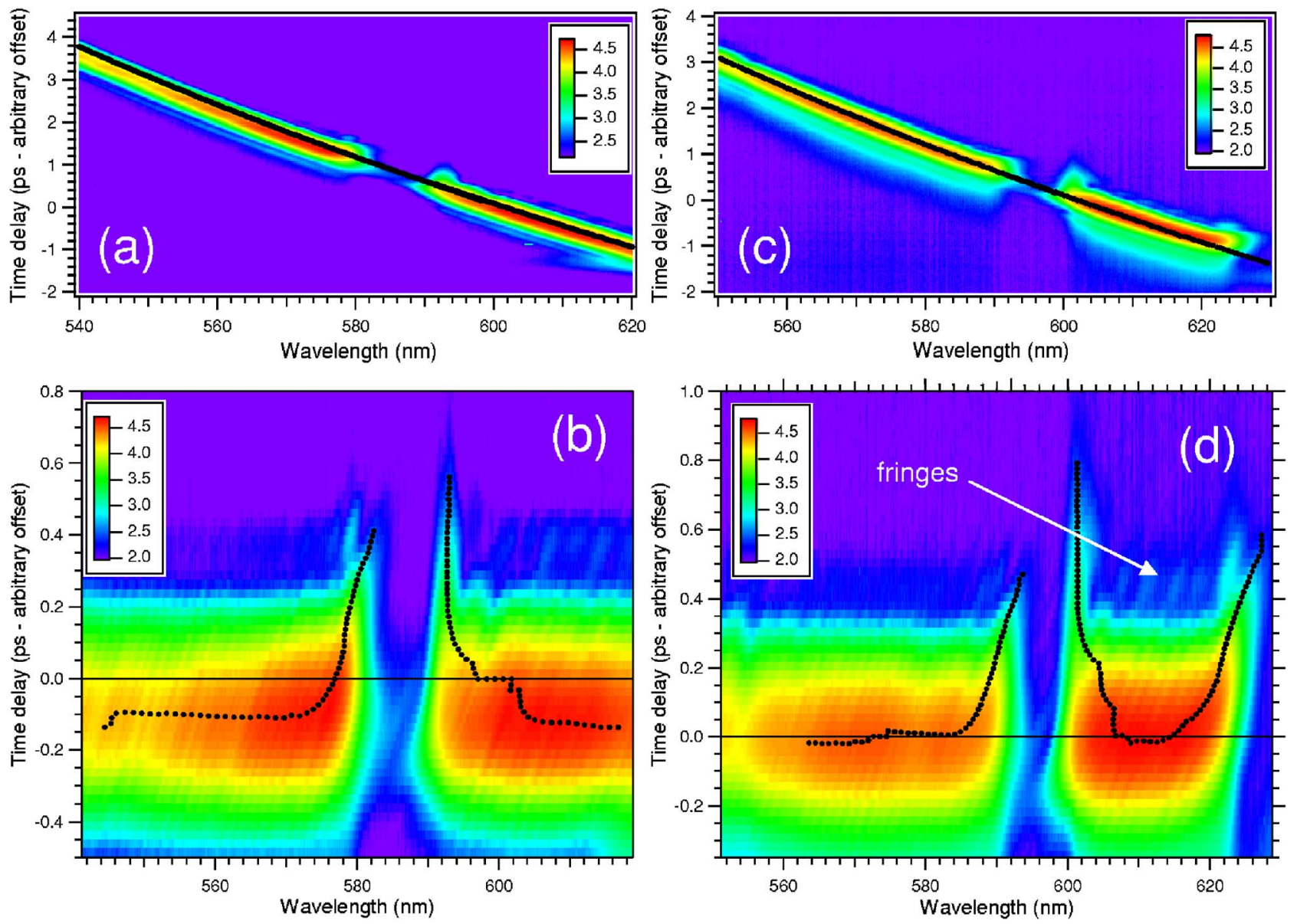

FIG. 5. (Color online) Time of flight TM-polarized dispersion data (on a log scale) in the spectral vicinity of one of the photonic band gaps, for structure $D$. (a) Uncorrected data—solid line shows measured dispersion for an unstructured waveguide. (b) Data corrected for the bulk waveguide dispersion. Peak wavelength positions of the gated intensity for successive scans are shown as black markers, and the temporal step size between spectra is 20 fs. Low-intensity fringing phenomena can be seen either side of the band gap, as indicated. (c), (d) Equivalent data for a characteristic band gap of structure $B$.

tion, in the vicinity of a typical PBG feature. In Fig. 5(b), this plot has been corrected for the measured GVD of the corresponding waveguide reference sample, in order to allow an unambiguous determination of any effects due to the PC. We see that the PC contribution to the time of flight is essentially independent of wavelength away from the spectral vicinity of the PBG, whereas a radical disruption of the GVD is seen around the band gap. In particular, we see the expected disappearance of the signal transmitted through the Kerr gate at wavelengths corresponding to the PBG, and also significant anomalies of the time of flight are seen at the band-gap edges, with detectable components of the continuum being delayed by up to $\sim 1$ ps. Similar effects are also observed at other characteristic PBG's of this sample (see Fig. 6) and, indeed, also for other samples of varying optical band-structure design, which we have examined; typically we observe "slowed light" with time delays extending to values of between 0.1 and $\sim 1$ ps.

Using the $2 \mathrm{D}$ plane-wave analysis techniques, as described above, it is also possible to produce quantitative models of the characteristic GVD and time-of-flight properties of our samples. By considering the gradient of the allowed photonic bands, $\omega(k)$, generated by the plane-wave model, it is possible to calculate the group velocity of guided light as a function of normalized frequency and, therefore, the expected time delay of components propagating in the vicinity of the PBG. In Fig. 6, the predicted time delay as a function of wavelength is compared to the complete experimental data for each of the structures $A-D$. The model shows the expected asymptotic time-delay behavior within the band gaps and also some subtle variations in the GVD behavior at the band-gap edges, which reflect differences in the dispersion characteristics of the various air and dielectric bands as shown in Fig. 4(a). The experimental data are generally seen to be in good agreement with the theory, although the rapid decrease in detected signal with increasing time delay at the band-gap edges prevents a useful comparison beyond delays of $\sim 1 \mathrm{ps}$. Analysis relating to the loss mechanisms associated with this issue is dealt with in the following section.

The PBG dispersion delay is seen not to be a strong function of incident angle in the current geometry. We are able to investigate this lack of directional dependence further by developing a universal model of the dispersion around the PBG's, based on a simple two-band approach. This is mainly relevant in the low-order photonic bands that we are consid- 

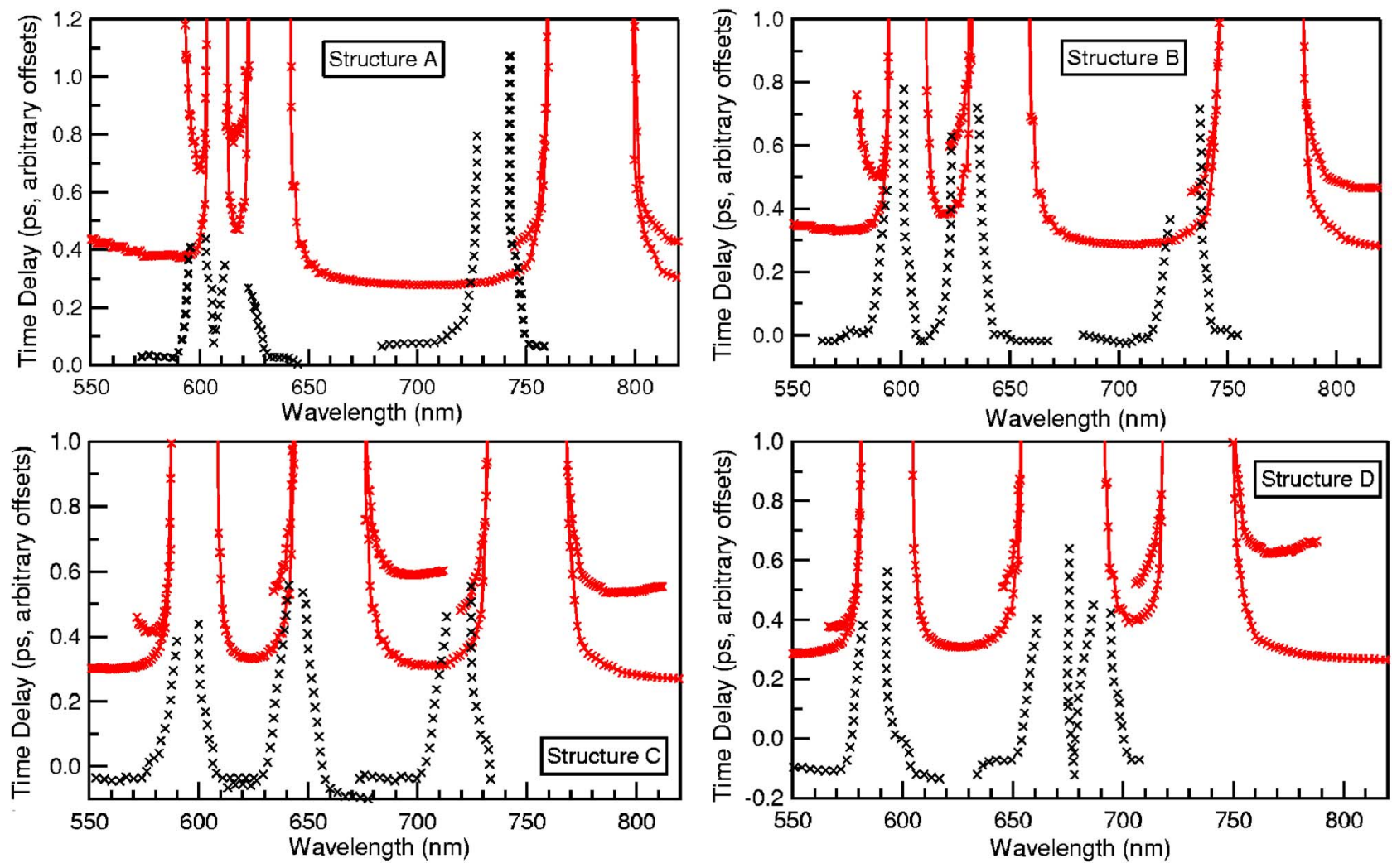

FIG. 6. (Color online) Time delay $T_{\mathrm{PC}}$ of slowed light as a function of wavelength, from experiment (black) and as inferred from the TM-polarized group velocities derived from our 2D plane-wave model for structures $A-D$ (red). The theoretical time delay is shifted by $200 \mathrm{fs}$ for clarity. The presence of additional branches of slowed light in the model, at longer time delays, is due to folding of bands in the plane-wave simulation.

ering here, in which mixing occurs from pairs of bands. The waveguide dispersion lines of these two bands (corresponding to the air and dielectric bands) in the immediate vicinity of the PBG are given by

$$
\begin{aligned}
& E_{1}=E_{0}+a k, \\
& E_{2}=E_{0}-a k,
\end{aligned}
$$

where $a=\hbar c / \eta$ and $\eta$ is the effective refractive index of guided modes. In the presence of strong scattering due to the interaction with the PC lattice, the perturbed branches of these bands, $E_{+}$and $E_{-}$, are mixed from the uncoupled states $E_{1}$ and $E_{2}$, giving

$$
E_{ \pm}=E_{0} \pm \sqrt{a^{2} k^{2}+\Delta^{2}}
$$

where the band-gap splitting equals $2 \Delta$. The group velocity dispersion can be calculated by differentiating this expression,

$$
v_{g}=\frac{1}{\hbar} \frac{d E}{d k}=\frac{c / \eta}{\sqrt{1+\frac{1}{\left[\left(\frac{E-E_{0}}{\Delta}\right)^{2}-1\right]}}},
$$

and the time of flight can then be simply inferred from $v_{g}$ and the sample dimensions. We thus arrive at a relationship for the time of flight, $T_{\mathrm{PC}}$, compared to the propagation time in the unpatterned waveguide, $T_{\mathrm{WG}}$,

$$
\left[\left(\frac{T_{P C}}{T_{W G}}\right)^{2}-1\right]\left[\left(\frac{E-E_{0}}{\Delta}\right)^{2}-1\right]=1
$$

This relationship holds for any band gaps formed by coupling two bands and shows that the increasing time of flight is inextricably linked to the proximity of the band edge in a universal fashion, which is independent of the orientation and design of the photonic crystal, except insofar as it modifies the band gap.

In Fig. 7, we compare the slowed-light components of the principal band gap, as calculated from the $2 \mathrm{D}$ plane-wave model, with the behavior predicted by our universal approach. For each of the structures $A-D$, careful fitting of the parameters $\Delta$ and $E_{0}$ gives excellent agreement between the two models, irrespective of the propagation direction through the structure. We note here the use of a global value of the refractive index $\eta$, corresponding to the gradient of the light line on which all the PBG features sit in Fig. 4.

We also frequently observe fringing effects in our timeof-flight data, whereby a series of low-intensity fringes is seen at later time delay after the main Kerr-gated pulses, as may be discerned from Figs. 5(b) and 5(d). We believe that these effects are caused by Fabry-Perot fringing between light which is multiply reflected by the interfaces at the edges of the PC pattern embedded in the waveguide, and this 

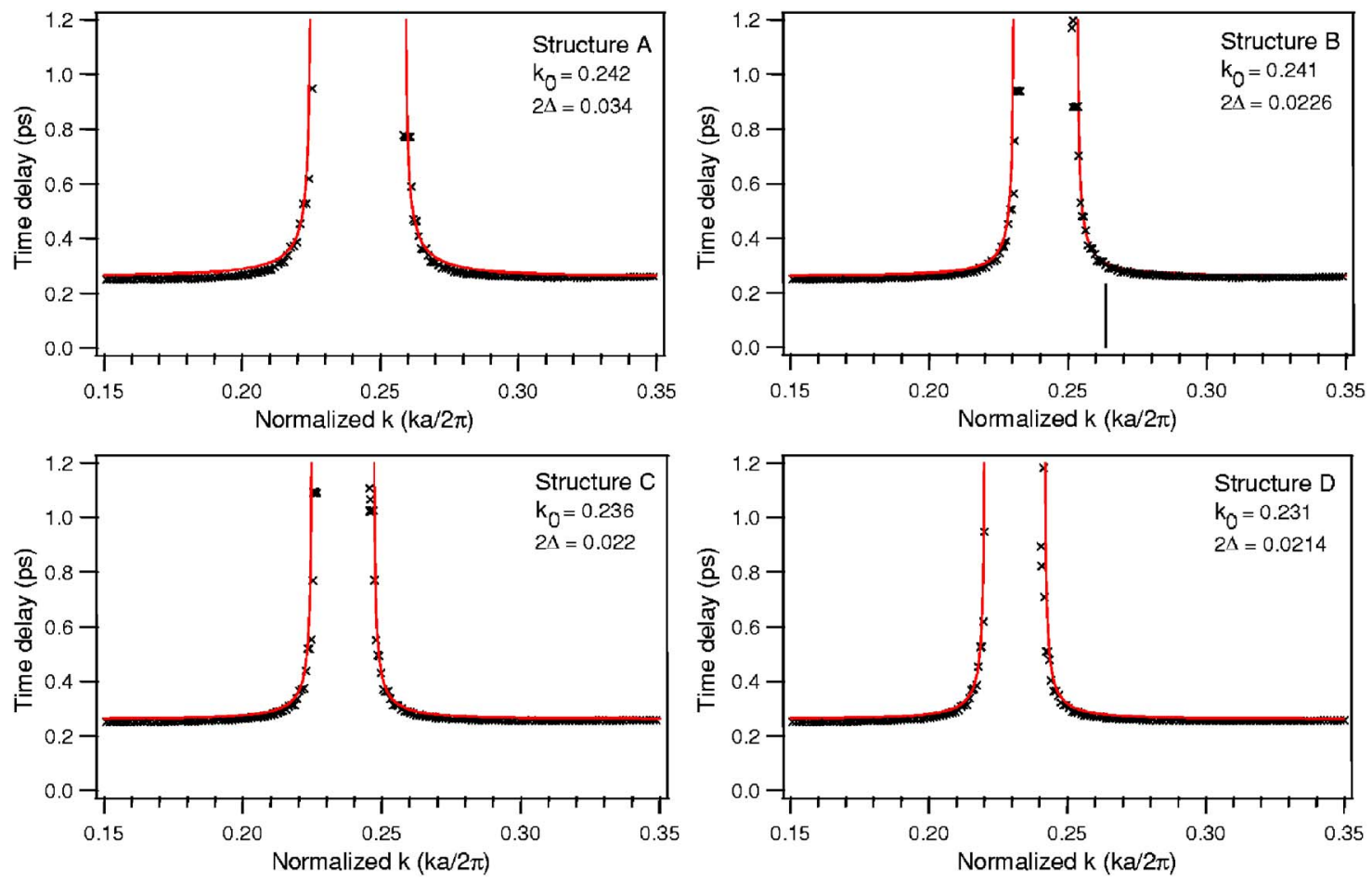

FIG. 7. (Color online) Comparison of slowed-light behavior predicted by a 2D plane-wave model (black crosses) with that derived from our universal two-band model (red lines) for the primary TM band gap in the case of each of structures $A-D$. The parameters used for the universal band model are given as insets to each graph; $k_{0}$ is the wave number corresponding to the center band-gap energy $E_{0}$.

is also predicted by theoretical analysis of GVD for PC structures of this kind [36]. These fringes in the time-of-flight plots are also seen to converge with each other and with the main gated light pulse at the spectral edges of the PBG's, further emphasizing that the delaying of light is due to the intrinsic GVD anomalies at the band gaps, rather than merely due to sample chirp or similar effects.

\section{ANALYSIS}

In order to place the observed time delay of components of "slowed light" into context, we quantitatively consider how the PC-enhanced anomalous GVD we observe compares, for example, to the dispersive effects seen in optical telecommunications fibers [37]. Taking a generalized view of our data in Figs. 5 and 6, time delays of up to 1 ps relative to the normalized dispersion of the waveguide medium are seen, within a wavelength range of approximately $\Delta \lambda$ $=5 \mathrm{~nm}$; this corresponds to an extensive sample dispersion of $0.2 \mathrm{ps} / \mathrm{nm}$ in the vicinity of the band-gap edges. Considering that the PC sample in each case is $46.5 \mu \mathrm{m}$ in length (150 rows $\times 310 \mathrm{~nm}$ pitch), this represents an anomalous dispersion per unit length of $\sim 3.5 \mathrm{ps} / \mathrm{nm}$ per $\mathrm{mm}$ of the structure. A simple estimation of the group velocity $\left(\nu_{g}\right)$ in the spectral vicinity of the PBG in our samples from the sample dimension along the direction of propagation $(l)$ and the time light takes to travel across the PC structure $(\tau)$ gives

$$
\nu_{g}=l / \tau \approx 0.15 \mathrm{c},
$$

corresponding to an effective refractive index of around $\eta$ $\sim 7$.
This demonstration of such unique properties as the wavelength-dependent enhanced effective refractive index and anomalous GVD effects reinforces the potential applications of PC structures as functional optical components. For example, our measurements indicate that, in principle, a 1 $\mathrm{cm}$ long PC structure could be used to compensate for the adverse optical dispersion effects in several kilometers of optical telecoms fiber [38]. Further judicious engineering and optimization of PC waveguide design [39] and the characteristic wavelength of operation should place this estimate at the modest end of the achievable range. Because of the presence of slowed-light components on both the high-energy and low-energy sides of the PBG in our samples, resulting in up-chirp and down-chirp spectral regions, it should also be possible to utilize both negative and positive dispersion compensation behavior of the PC depending on the application requirements.

In Fig. 8, the intensity of gated signal, as a function of the time delay, is shown for each of the PBG features, taking the well-spaced band gaps in structure $C$ as an example. By plotting the logarithm of intensity, we highlight the expected exponential decay behavior, although a noticeable deviation is sometimes seen at longer time delays, where the signal intensity decreases towards the limit of experimental detection. Of interest is the different attenuation behavior of the slowed-light intensity at the dielectric- and air-band sides of the band gaps. In particular, it is expected that slowed-light components propagating at the air-band edge will be attenuated more rapidly as a function of time delay than in the dielectric band. This is due to the different distributions of the optical field within the PC structure, with the air band 

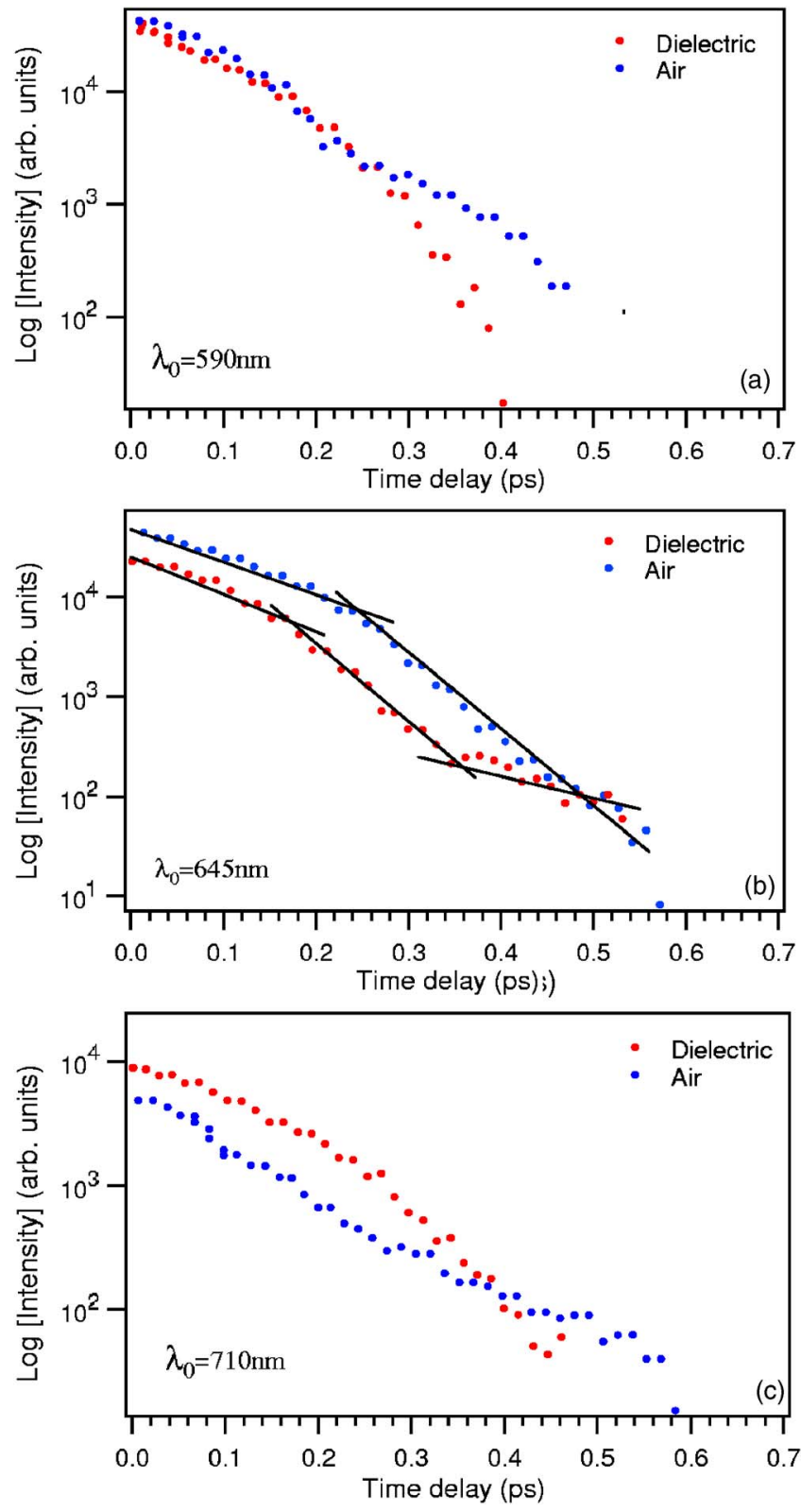

FIG. 8. (Color online) Gated intensity (log scale) as a function of time delay, extracted from the time-of-flight measurements, for each of the three TM band-gap features of structure $C$ (see Fig. 3). Both dielectric-band and air-band sides of each band gap are plotted. Guide lines have been added to (b) to demonstrate the increase in attenuation at longer delay times.

suffering greater up-scattering losses on account of the higher concentration of the optical field to the air holes of the structure [2]. However, the measured behavior is more complicated than this.

In order to better understand and quantify the behavior of slowed-light propagating at the band-gap edges, we need to additionally consider the effect of scattering losses in this particular situation. Koenderink and Vos [40] have recently modeled the light propagating through a two-dimensional PC in terms of scattering losses due to structural inhomogenieties. The uncertainty in both hole radius and hole position can have a significant detrimental effect upon the photonic
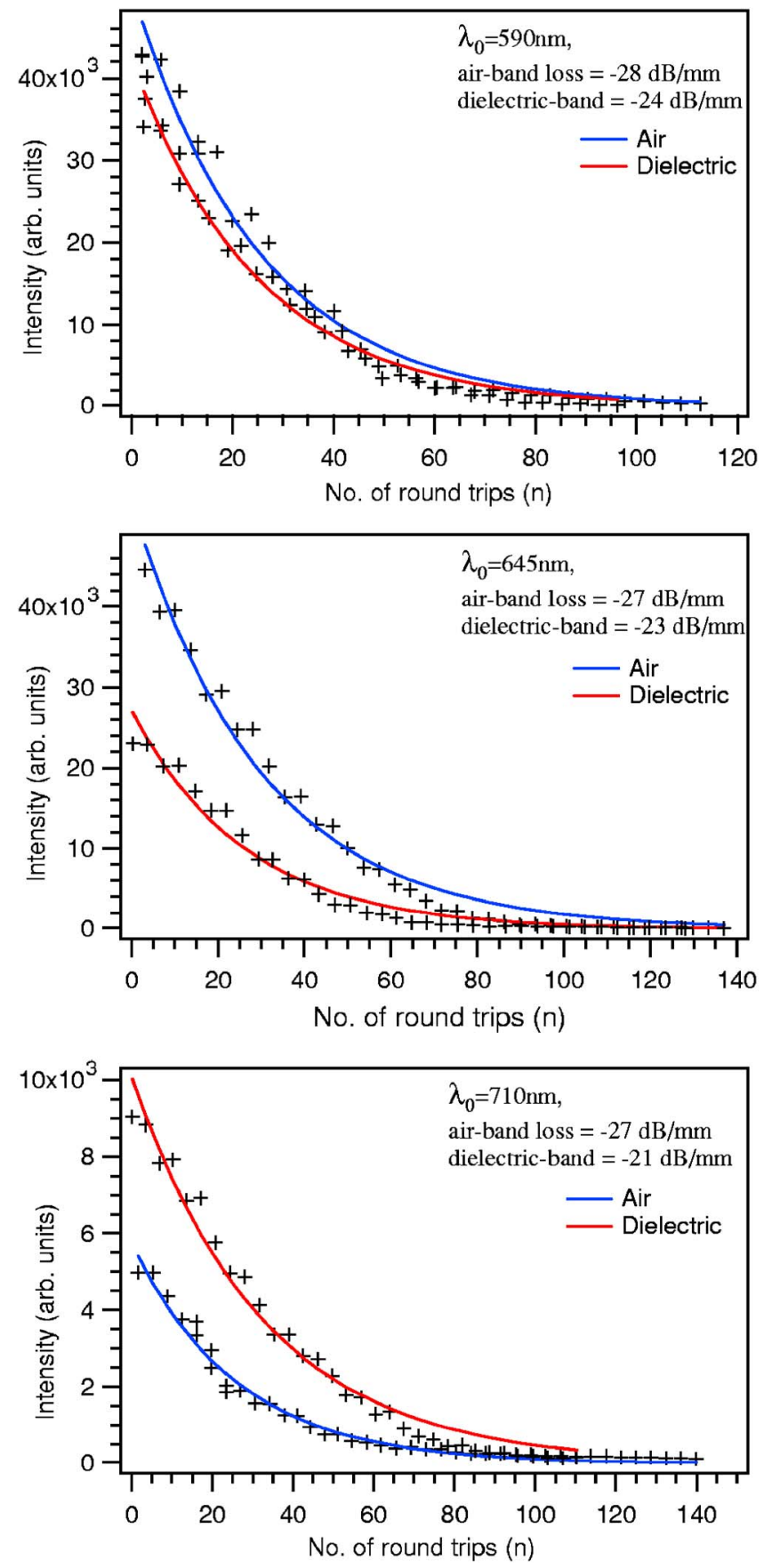

FIG. 9. (Color online) Gated intensity (linear scale) of bandedge light as a function of the number of round-trips between holes spaced by the pitch $a=310 \mathrm{~nm}$. Best fits shown (red line for dielectric band, blue line for air band) are obtained from a least-squares algorithm based on Eq. (7). The loss coefficient of slowed light is extracted for both the air- and dielectric-band edges in each case.

band-gap properties and scattering centers introduced by these factors can be used to derive a linear-loss coefficient, based on a single pass of the structure. In the case of light which propagates in the spectral vicinity of the band gap, multiple-reflection and resonant-scattering effects produce an increase in the optical path, which manifests itself in the phenomenon of slowed light. In physical terms, this model may alternatively be imagined in terms of delayed light com- 
ponents having undergone multiple resonant scattering during propagation through the $\mathrm{PC}$ at the band-gap edges, where the scattering cross section from defects is also greatest. Hence, those components with the greatest time delay will have undergone the greatest number of scattering events and passed the greatest physical distance across the PC medium. Upon each successive round-trip between scattering centers (i.e., the sample pitch), the losses will scale as the $n$th power of the number of round-trips; hence, the gated intensity is expected to drop off very rapidly as a function of time delay. This basic model is therefore qualitatively consistent with the observed behavior, as shown in Figs. 5 and 8. This behavior can be described as

$$
I=I_{0}(1-\alpha)^{n},
$$

where $I$ is the gated intensity, $I_{0}$ is the gated intensity measured for zero time delay, and $\alpha$ is the fractional loss per round-trip. Taking the sample pitch of $a=310 \mathrm{~nm}$ as the scattering length, $n$ is the number of round-trips between scattering centers, given by the expression

$$
n=\frac{c T_{P C}}{a \eta},
$$

where $c$ is the speed of light, $T_{P C}$ is the time of flight as previously defined, and $\eta$ is the effective refractive index. In Fig. 9, the data for structure $C$ is shown as gated intensity against number of round-trips, having taken into account the sample pitch and the refractive index of the waveguide. We find that this basic model yields a satisfactory fit to the data for each of the slowed-light branches under scrutiny, as shown in Fig. 8. This ray optics approach has limitations given that the sample pitch is of the same order of the wavelength of the propagating light; however, it does enable a quantitative estimation of the characteristic losses of the slowed light, inferred from the parameter $\alpha$. In this way we estimate that modes propagating at the dielectric-band edge had losses of $-22 \pm 2 \mathrm{~dB} / \mathrm{mm}$, whereas the air-band modes are found to have losses of $-27 \pm 1 \mathrm{~dB} / \mathrm{mm}$. To a first approximation, these levels of attenuation confirm the expected behavior between the two modes and we find that light propagating in the vicinity of the band edges travels only $\sim 30$ round-trips between holes on average before scattering out of the photonic crystal waveguide. This compares to the insignificant loss of the light propagating through 150 holes, for wavelengths away from the band gap, demonstrating how the enhanced scattering cross section per hole is a sensitive function of wavelength. In Fig. 8(b), guidelines indicate how the attenuation is generally seen to increase at longer delay times and, interestingly, we also observe less attenuation in the air band at longer times. This is possibly due to memory effects in the multiple scattering of light, in which photons revisit scattering centers [41]. We note that, although our experiments demonstrate that impressively high anomalous dispersions are possible at the band-gap edges, the losses of such propagating modes are currently high within the current generation of our PC waveguide structures.

\section{CONCLUSIONS}

In this paper, we have clearly and directly observed signatures of slowed light due to the photonic band-gap influence in low-loss 2D photonic-crystal waveguides with timeof-flight techniques utilizing a femtosecond white-light supercontinuum and an optical Kerr gate. Broadband transmission measurements show our structures to possess verylow-loss guiding properties. Slowed-light components with time delays of up to $1 \mathrm{ps}$, corresponding to an anomalous dispersion of $\sim 3.5 \mathrm{ps} / \mathrm{nm}$ per $\mathrm{mm}$ of sample, have been tracked and characterized with the aid of suitable 2D planewave theoretical models of the photonic band structure. By considering how the intensity of gated slowed light decreases as a function of time delay and by using a simple multipleresonant-scattering model, we are able to estimate the characteristic losses of modes which are guided in the spectral proximity of the photonic band gaps as being in the range of -20 to $-30 \mathrm{~dB} / \mathrm{mm}$. We propose that the techniques reported here represent a very powerful tool for the further characterization and optimization of photonic-crystal structures and designs, including issues relating to losses of optical modes propagating at the band-gap edges, and for the development of highly engineered integrated optical circuits based on silicon-compatible planar waveguide platforms.
[1] E. Yablonovitch, Phys. Rev. Lett. 58, 2059 (1987).

[2] J. D. Joannopoulos, R. D. Meade, and J. N. Winn, Photonic Crystals (Princeton University Press, Princeton, NJ, 1995).

[3] E. Yablonovitch, J. Opt. Soc. Am. B 10, 283 (1993).

[4] P. St. J. Russell, Phys. Rev. A 33, 3232 (1986).

[5] R. Zengerle, J. Mod. Opt. 34, 1589 (1987).

[6] M. C. Netti, M. D. B. Charlton, G. J. Parker, and J. J. Baumberg, Appl. Phys. Lett. 76, 991 (2000).

[7] D. J. Norris and Y. A. Vlasov, Adv. Mater. (Weinheim, Ger.) 13, 371 (2001).

[8] A. Yariv, Y. Xu, R. K. Lee, and A. Scherer, Opt. Lett. 24, 711 (1999).

[9] D. Labilloy, H. Benisty, C. Weisbuch, T. F. Krauss, R. M. De La Rue, V. Bardinal, R. Houdre, U. Oesterle, D. Cassagne, and
C. Jouanin, Phys. Rev. Lett. 79, 4147 (1997).

[10] R. T. Neal, M. E. Zoorob, M. D. Charlton, G. J. Parker, C. E. Finlayson, and J. J. Baumberg, Appl. Phys. Lett. 84, 2415 (2004).

[11] S. G. Johnson, S. Fan, P. R. Villeneuve, J. D. Joannopoulos, and L. A. Kolodziejski, Phys. Rev. B 60, 5751 (1999).

[12] M. E. Zoorob, M. D. B. Charlton, G. J. Parker, J. J. Baumberg, and M. C. Netti, Nature (London) 404, 740 (2000).

[13] G. Vecchi, J. Torres, D. Coquillat, M. Le V. d' Yerville, and A. M. Malvezzi, Appl. Phys. Lett. 84, 1245 (2004).

[14] S. Noda, A. Chutinan, and M. Imada, Nature (London) 407, 608 (2000).

[15] M. D. Tocci, M. Scalora, M. J. Bloemer, J. P. Dowling, and C. M. Bowden, Phys. Rev. A 53, 2799 (1996). 
[16] K. J. Vahala, Nature (London) 424, 839 (2003).

[17] A. Imhof, W. L. Vos, R. Sprik, and A. Lagendijk, Phys. Rev. Lett. 83, 2942 (1999).

[18] H. Gersen, J. P. Korterik, N. F. van Hulst, and L. Kuipers, Phys. Rev. E 68, 026604 (2003).

[19] H. Kosaka, T. Kawashima, A. Tomita, T. Tamamura, T. Sato, and S. Kawakami, Appl. Phys. Lett. 74, 1370 (1999).

[20] M. Notomi, K. Yamada, A. Shinya, J. Takahashi, C. Takahashi, and I. Yokohama, Phys. Rev. Lett. 87, 253902 (2001).

[21] Y. A. Vlasov, S. Petit, G. Klein, B. Hönerlage, and C. Hirlimann, Phys. Rev. E 60, 1030 (1999).

[22] G. von Freymann, S. John, S. Wong, V. Kitaev, and G. A. Ozin, Appl. Phys. Lett. 86, 05308 (2005).

[23] K. Inoue, N. Kawai, Y. Sugimoto, N. Carlsson, N. Ikeda, and K. Asakawa, Phys. Rev. B 65, 121308 (2002).

[24] H. Gersen, E. M. H. P. van Dijk, J. P. Korterik, N. F. van Hulst, and L. Kuipers, Phys. Rev. E 70, 066609 (2004).

[25] H. Gersen, T. J. Karle, R. J. P. Engelen, W. Bogaerts, J. P. Korterik, N. F. van Hulst, T. F. Krauss, and L. Kuipers, Phys. Rev. Lett. 94, 073903 (2005).

[26] J. Takeda, K. Nakajima, S. Kurita, S. Tomimoto, S. Saito, and T. Suemoto, Phys. Rev. B 62, 10083 (2000).

[27] S. Kinoshita, H. Ozawa, Y. Kanematsu, I. Tanaka, N. Sugimoto, and S. Fujiwara, Rev. Sci. Instrum. 71, 3317 (2000).

[28] M. C. Netti, C. E. Finlayson, J. J. Baumberg, M. D. B. Charlton, M. E. Zoorob, J. S. Wilkinson, and G. J. Parker, Appl. Phys. Lett. 81, 3927 (2002).

[29] K. Sakoda, Optical Properties of Photonic Crystals (Springer,
Heidelberg, 2001).

[30] M. D. B. Charlton, S. W. Roberts, and G. J. Parker, Mater. Sci. Eng., B 49, 155 (1997).

[31] V. N. Astratov, R. M. Stevenson, I. S. Culshaw, D. M. Whittaker, M. S. Skolnick, T. F. Krauss, and R. M. De La Rue, Appl. Phys. Lett. 77, 178 (2000).

[32] C. J. M. Smith, H. Benisty, S. Olivier, M. Rattier, C. Weisbuch, T. F. Krauss, R. M. De La Rue, R. Houdre, and U. Oesterle, Appl. Phys. Lett. 77, 2815 (2000).

[33] M. D. B. Charlton, M. E. Zoorob, M. C. Netti, N. Perney, G. J. Parker, P. Ayliffe, and J. J. Baumberg, Microelectron. J. 36, 277 (2005).

[34] L. C. Andreani, Phys. Status Solidi B 234, 139 (2002).

[35] A. Taflove, Advances in Computational Electrodynamics; The Finite-Difference Time-Domain Method (Artech House, Norwood, 1998).

[36] M. E. Zoorob (private communication).

[37] A. Ghatak and K. Thygarajan, Introduction to Fibre Optics (Cambridge University Press, Cambridge, England, 1998).

[38] J. C. Knight, J. Arriaga, T. A. Birks, A. Ortigosa-Blanch, W. J. Wadsworth, and P. St. J. Russell, IEEE Photonics Technol. Lett. 12, 807 (2000).

[39] M. L. Povinelli, S. G. Johnson, and J. D. Joannopoulos, Opt. Express 13, 7145 (2005).

[40] A. F. Koenderink and W. L. Vos, J. Opt. Soc. Am. B 22, 1075 (2005).

[41] M. Tomita, Phys. Rev. B 45, 1045 (1992). 